\title{
How we approach the diagnosis and management of complex lymphatic anomalies
}

\author{
Kiersten Ricci ${ }^{1}$ and Ionela Iacobas ${ }^{2}$ \\ ${ }^{1}$ Cincinnati Children's Hospital Medical Center \\ ${ }^{2}$ Baylor College of Medicine
}

November 23, 2020

\begin{abstract}
Complex lymphatic anomalies are congenital diseases of the lymphatic circulation system that are associated with significant morbidity and early mortality. While guidelines for the comprehensive evaluation of the complex lymphatic anomalies were recently published, the diagnostic approach and medical management are not standardized. The current manuscript presents the clinical features of 4 complex lymphatic anomalies: Gorham-Stout disease, generalized lymphatic anomaly, kaposiform lymphangiomatosis, and central collecting lymphatic anomaly. We also offer three cases from the authors' practice and our views on diagnostic testing and disease management including supportive care, medical therapies and other interventions.
\end{abstract}

\section{INTRODUCTION}

Complex lymphatic anomalies (CLA) are rare conditions that occur due to embryonal errors in lymphangiogenesisis and are associated with significant morbidity. CLA include Gorham-Stout disease, generalized lymphatic anomaly, kaposiform lymphangiomatosis, and central collecting lymphatic anomaly [1]. These conditions have phenotypic heterogeneity as well as overlapping symptoms, imaging features and complications of these disorders. Management of complex lymphatic anomalies is often challenging, requiring multimodal and interdisciplinary approach.

\section{Gorham-Stout Disease}

Gorham-Stout disease (GSD) or "vanishing bone disease" is a multifocal lymphatic malformation (LM) that classically affects bone but may also involve soft tissue and viscera. GSD is characterized by progressive osteolysis and proliferation of lymphatic vessels, leading to the destruction and absorption of bone. The most commonly affected bones include the vertebrae, ribs, skull, jaw, and clavicle [2]. Multiple bones may become involved, often in a contiguous distribution. In addition to frequently causing pain and functional impairment, GSD may cause significant morbidity. Complications may include pathologic fractures, pleural and pericardial effusions, CSF leaks, deformity and neurologic deficits secondary to osteolysis of the spine and/or skull. Computed tomography (CT) and magnetic resonance imaging (MRI), along with a thorough medical history, are usually sufficient for diagnosis. On imaging, evidence of destruction of the bone cortex is critical for diagnosis [3]. Histology reveals abnormal, dilated lymphatic channels with endothelial cells that stain positive for the lymphatic markers, PROX-1 and D2-40, as well as increased osteoclast activity in bone [4]. No causative genetic mutations have been identified.

\section{Generalized Lymphatic Anomaly}

Generalized lymphatic anomaly (GLA) is characterized by multifocal LM that involves the bones, viscera, thoracic and abdominal cavities. Although GLA has similar areas of anatomic involvement and complications as GSD, the disorder is distinctively different. In contrast to GSD, the lytic bone lesions in GLA are confined 
to the medullary cavity and do not cause destruction of bone cortex. GLA favors the appendicular skeleton and typically involves multiple body sites in a non-contiguous pattern [1,2]. Lesions in the spleen and liver are commonly seen on imaging [5]. Pathology appears similar to that of GSD with exception of bone cortex disruption [4]. Somatic PIK3CA (phosphatidylinositol-4,5-bisphosphate 3-kinase, catalytic subunit alpha) mutations were recently discovered in individuals with GLA [6].

\subsection{Kaposiform Lymphangiomatosis}

Kaposiform lymphangiomatosis (KLA) is an aggressive lymphatic anomaly that is associated with significant morbidity and high mortality. KLA most commonly involves the thoracic cavity, bone and spleen [7]. Clinical presentation is typically severe, particularly at a young age, and progression occurs without treatment. Complications include organ compromise, pleural and pericardial effusions, ascites, pain, and functional issues. KLA is associated with a life-threatening coagulopathy characterized by severe hypofibrinogenemia, thrombocytopenia and bleeding [8]. Effusions and ascites are often hemorrhagic. Defining histopathological features are abnormal lymphatic channels with focal areas of spindle-shaped "kaposiform" endothelial cells, positive for D2-40 and PROX-1 [7]. Platelet trapping is also seen in KLA, suggesting that the pathogenesis of the coagulopathy is similar to Kasabach-Merritt phenomenon (KMP) occurring in kaposiform hemangioendothelioma (KHE) and tufted angioma (TA) [9]. Recently, RAS mutations were identified in affected tissue of patients with KLA [10].

\subsection{Central Conducting Lymphatic Anomaly}

Central conducting lymphatic anomaly (CCLA) encompasses disorders that are caused by dysfunction of the thoracic duct and/or cisternae chylae with subsequent reflux and leakage of lymphatic fluid, most commonly into the lungs and/or abdomen [1]. Pleural and pericardial effusions, ascites and generalized edema are common and can result in organ dysfunction, protein loss and infections. Although medical history, physical exam and standard imaging may be suggestive of CCLA, definitive diagnosis requires lymphangiography demonstrating enlargement of lymphatic channels (lymphangiectasia), lymphatic fluid reflux and/or failure to empty into the thoracic duct or the subclavian vein at the thoracic duct outlet. Dynamic magnetic resonance lymphangiography may also be used for diagnosis and to help guide treatment but is not widely available $[11,12]$.

Treatment is largely supportive. Medical therapies have historically been limited to compression garments and replacement of albumin and immunoglobulins. Disease-modifying agents such sirolimus have been used but response is unclear, although a pathogenic EPHB4 (ephrin type-B receptor 4) variant was recently discovered, suggesting a potential role for mTOR or MAPK/MEK inhibitors [13]. Surgical procedures such as re-implantation of the thoracic duct or embolization of refluxing channels may also be a treatment consideration.

Table 1 provides a summary of the clinical features of these CLA.

\section{MANAGEMENT PRINCIPLES FOR COMPLEX LYMPHATIC ANOMALIES}

\subsection{Diagnosis}

In the past, the term "hemangioma" was used to represent all benign vascular lesions regardless of etiology, disease course or pathology. Based on clinical characteristics, radiologic imaging and pathologic findings, the International Society for the Study of Vascular Anomalies (ISSVA) created a standardized classification system in 1996 which broadly categorized vascular anomalies as tumors and vascular malformations. The ISSVA classification system was most recently updated in 2018 and can be accessed atwww.issva.org/classification.

As we will illustrate in the following 3 cases, initial presentation of CLA is variable, and the provider needs to have a high index of suspicion for and familiarity with these conditions in order to provide timely diagnosis and appropriate management.

Radiologic evaluation should include imaging of the chest, abdomen, pelvis and total spine with CT and/or MRI. If suspicion for GSD, skull imaging is indicated. Skeletal bone survey may be used to focus imaging 
needs. MR lymphangiogram can also be helpful in certain situations [14].

Laboratory investigation should include complete blood count with differential, D-dimer, fibrinogen, prothrombin time (PT), partial thromboplastin time (PTT), albumin and total protein, immunoglobulins, liver and renal function tests, electrolytes and bone turn-over markers (alkaline phosphatase, C-telopeptide, etc.).

The extent and characteristics of the bony lesions, distribution of viscera involvement, laboratory findings generally will provide criteria for final diagnosis, frequently without a biopsy. In situations in which the diagnosis is unclear despite medical history, imaging and laboratory findings, biopsy of a soft tissue lesion is recommended over bone biopsy. Biopsy of ribs and vertebrae should be avoided as these can result in pleural effusions and CSF leaks $[15,16]$. In patients with KLA, biopsy may result in significant bleeding and worsen the underlying coagulopathy. Because mutations in CLA are somatic, genetic testing of the affected tissue, not peripheral blood, should be considered to guide therapy.

\subsection{Potential Complications}

Patients with CLA suffer from pain and functional impairment, psychosocial stress and decreased quality of life (QOL). CLA have distinctive, yet often overlapping complications as below.

- Lytic bone lesions. Bone lesions may cause pain and predispose to pathologic fractures. Pathologic fractures tend to occur most frequently in GSD because of contiguous bone involvement and cortical bone destruction. Lytic bone lesions usually do not occur in individuals with CCLA.

- Coagulopathy. Patients with KLA develop severe thrombocytopenia and hypofibrinogenemia, frequently causing bleeding complications. GSD, GLA, and CCLA are not associated with coagulopathy.

- Chylous effusions. Accumulation of chylous pleural and pericardial effusions, along with lymphatic ascites, may be severe and lead to cardiopulmonary compromise or death. Chylous effusions may occur in patients with all 4 CLA. However, effusions are usually hemorrhagic in individuals with KLA.

- Protein losses. Loss of proteins and lymphocytes, sometimes in massive quantities, may occur when lymphatic fluid accumulates in effusions, particularly when surgically drained. Hypogammaglobulinemia and lymphopenia may cause immunocompromise. Hypoalbuminemia leads to decreased oncotic pressure resulting in generalized edema while other protein losses may impair growth. Protein losses may occur in all 4 CLA but occurs most frequently with CCLA.

\subsection{Treatment}

CLA have no known cure. During the last few decades, scientific research, along with genetic and technological advancements, has enhanced our knowledge of the clinical behavior, pathology, genetics and molecular biology of vascular anomalies. These insights have changed the paradigm in how patients with CLA are managed, focusing on prevention, reduction of complications, and improvement of QOL.

\subsubsection{Medical Management}

Medical management of CLA involves supportive care and targeted drug therapy. Improved understanding of pathogenesis and genetic etiology for lymphatic anomalies lead to use of mTOR inhibitors such as sirolimus and everolimus, bisphosphonates (ex. zoledronic acid) and more recently, MAPK/MEK inhibitors (ex. trametinib). PIK3CA inhibitors are also a potential treatment option in the future. Multiple case series and reports describe treatment algorithms of different institutions, but there is no consensus on indications for starting medication, initial dosing, dosing adjustments, frequency of monitoring or length of therapy. Since no universal guidelines exist, we offer, in Table $\mathbf{2}$, our institutional protocols for these medications.

\subsubsection{Endovascular Interventions}

Expertise of Interventional radiology (IR) and other interventional specialties (ex. cardiology, neurosurgery) is frequently necessary in the multimodal treatment approach of CLA. IR expertise is needed for lymphoscintigraphy and MR lymphangiography. Lymphoscintigraphy provides dynamic information on flow of lymphatic fluid but lacks spatial resolution and anatomic details. MR lymphangiography provides dynamic and anatomical information of the lymphatic vasculature and can also allow for therapeutic intervention. 
Progressive accumulation of contrast material followed by slow dispersion into the pleural and/or pericardial spaces helps identify the source of effusions. If a specific area of leakage is found, then embolization with ethiodized oil (Lipiodol) injection is a therapeutic option. Sclerotherapy is also a treatment option for macrocystic and microcystic LM. Sclerotherapy is used to reduce size of LM, improve pain, and decrease complications like frequent infections or bleeding into the cysts.

\subsubsection{Surgical Interventions}

Surgical interventions may be supportive or targeted towards the lymphatic disease. Supportive procedures include surgical stabilization of fractures and chest tube drainage. Examples of targeted surgical interventions include laser ablation of lymphatic vesicles, excisional debulking, lymphatico-venous anastomosis and thoracic duct re-implantation. If applicable, medical therapies should be attempted prior to surgical intervention. To illustrate, placing hardware to stabilize bone affected by CLA often results in hardware failure, lymphatic fluid or CSF leakage, and post-operative infection. Surgery may be avoided by first treating with disease-modifying agents such as sirolimus and zoledronic acid.

\subsection{Monitoring}

Monitoring for potential complications related to the natural course of CLA, as well as to those secondary to procedures and medical therapies, is essential. Although guidelines for the initial comprehensive evaluation were published recently [14], no consensus statements exist for monitoring with physical examination, imaging, or laboratory testing after diagnosis or while on specific medical treatments.

\section{CASES}

\subsection{Case 1: Gorham-Stout Disease}

An 8-year-old male presented with chronic headaches and history of 5 episodes of meningitis caused by Streptococcus viridans orpneumoniae. Past medical history included refractory immune thrombocytopenia (ITP) which resolved after splenectomy. He was diagnosed with "lymphangiomatosis" at an outside institution and initially treated with propranolol and vincristine, and then briefly with sirolimus. All medications were stopped when he developed meningitis. After additional episodes of meningitis, he was referred to our institution. He suffered from daily debilitating headaches initially thought to be secondary to past meningitis infections. MRI of the brain and conventional CT of the head were not able to identify the source of his recurrent meningitis. CT cysternogram demonstrated an active CSF leak at the central skull base due to osseous erosion of lateral walls of the sphenoid sinuses (Figure 1A-D) . Comprehensive imaging showed extensive spinal involvement (Figure 1E), lytic humeri lesions and a mediastinal LM. Since blood patch was unable to be performed due to the location and size of the dura mater defect, patient was initiated on interferon and zoledronic acid. After two years meningitis recurrence, interferon was stopped and oral sirolimus was started.

\subsubsection{Diagnostic Considerations}

In our case, the patient's diagnosis of GSD was made based on a combination of symptoms and imaging findings. Chronicity of his symptoms over several years and absence of a primary tumor made a malignant process highly unlikely. While osteolytic lesions do occur in the skull base in Langerhans cell histiocytosis (LCH), LCH bone lesions more commonly affect the calvarium and the temporal bone within the skull base. CSF leaks may also occur with LCH but are more frequently encountered in GSD. Furthermore, the presence of a mediastinal LM is suggestive of CLA.

Historically, lymphangiomatosis was an umbrella "diagnosis" that encompassed all CLA. The term, lymphangiomatosis, should be avoided since it is non-specific and could delay appropriate care or inadequately address potential complications. While our patient was diagnosed with "lymphangiomatosis" early in life, the exact type of CLA was not delineated because of diagnostic challenges. Sometimes the initial clinical presentation does not offer all the clues for an accurate diagnosis, especially in young children. Maintaining a high index of suspicion and re-analyzing the diagnosis when new complications occur is important. 
Identification of the area of bone destruction and dura matter defect can be difficult. The exact mechanism in which CSF leaks develop in GSD is unclear but is hypothesized to occur when the lymphatic malformation destroys the bone cortex of vertebrae or skull base and then infiltrates into dura mater. Spinal instability secondary to significant vertebrae or skull base destruction may also result in CSF leak.

Our patient's case was complicated by recurrent meningitis infections, a known complication of patients with GSD and skull base defects [17-19]. Although the etiology of recurrent meningitis isn't completely understood, the highest risk of infection appears to be with osteolytic nasotemporal lesions [17]. In our patient, oropharyngeal bacteria presumably translocated from the sphenoid sinus through porous eroded bone and into the CSF space through the dural mater defect. Our patient was also asplenic, predisposing him to infection with encapsulated bacteria. Comprehensive immunologic testing did not identify a primary immunodeficiency.

Although our patient had thrombocytopenia, his platelet count normalized after splenectomy, consistent with ITP. Furthermore, he had normal fibrinogen levels and no hemorrhagic effusions, arguing against a diagnosis of KLA.

\subsubsection{Management Considerations}

Management of our patient presented a challenge due to disease location and high recurrence rate of meningitis. Upon the identification of the area of CSF leak, interventional closure of the dura mater defect is ideal in order to prevent life-threatening meningitis events as well as to improve symptoms. However, in our patient, surgical closure was not an option given the location and size of the dura matter defect. Thus, medical therapy targeted towards GSD management became the priority.

The risk of using systemic sirolimus was initially considered too high due to its immunosuppressive effects in the setting of our patient's recent life-threatening infections. Weekly subcutaneous pegylated interferon alpha $2 \mathrm{~b}$ was initiated given its reported beneficial effect in GSD [20]. Interferon alpha 2b, only available in injectable form, became less preferentially used as first line therapy when sirolimus demonstrated efficacy in treating GSD. Interferon alpha $2 \mathrm{~b}$ has a black box warning for potential development of spastic diplegia in patients younger than 3 years (our patient was older) and can cause flu-like symptoms such as myalgia, headaches, fatigue, and low-grade fever within 24 hours after administration.

In order to inhibit osteoclastic activity and try to prevent further bone destruction, our patient also received bisphosphonate therapy with zoledronic acid. The combination of mTOR inhibitor and bisphosphonates has been described to have good effect in complex lymphatic anomalies with bone lytic lesions [21].

Our patient's headaches resolved on interferon alpha $2 \mathrm{~b}$ and bisphosphonate therapy. Repeat cysternogram showed improved intracranial hypotension and substantially smaller dura mater defect, although not completely closed. After two years without any new episodes of meningitis, we transitioned to sirolimus and zoledronic acid. Some of his lytic bone lesions have improved, while the remainder are stable. Although non-curative, his treatment regimen has not only stopped the disease progression but also lessened his CSF leak and intracranial hypotension, resolved his headaches and improved his QOL.

\subsection{Case 2: Generalized Lymphatic Anomaly}

A 12-year-old male presented to the emergency room with fever, tachycardia, hypotension, and difficulty breathing. Chest x-ray showed large right-sided pleural effusion causing midline shift (Figure 2A) . Broadspectrum antibiotics were started, and a chest tube was urgently placed, which drained milky white pleural fluid. Pleural fluid cultures grew Streptococcus pneumoniae. Chest CT demonstrated multiple lytic lesions involving the ribs. Medical history revealed that the patient had a "cyst" removed from his back when he was a toddler.

\subsubsection{Diagnostic Considerations}

This case highlights the importance of an accurate diagnosis and the need for improved familiarity and understanding of vascular anomalies by providers as well as by families and patients. In our patient's case, 
the excised "cyst" was a LM, which was confirmed upon review of his prior pathology. While an isolated LM would not raise suspicion for a CLA, this history was an important clue in his final diagnosis. The combination of soft tissue LM, lytic lesions of the ribs and development of a large chylothorax raises suspicion for CLA. Given concern for GLA, MRI of the abdomen, pelvis and spine revealed lesions in the pelvic bone, multiple vertebrae, and spleen as well as the presence of multiple mesenteric and retroperitoneal cysts (Figure 2B) . No cortical bone destruction was evident on imaging. Chest imaging with CT or MRI is also indicated in the workup of possible GLA, which our patient had after chest tube placement. Echocardiogram did not demonstrate pericardial effusion. Given the constellation of these findings, he was diagnosed with GLA.

Bone lesions are a common finding in pediatric and adult patients. Most bone lesions are benign tumors in children. Plain film is the primary modality in the initial evaluation of osseous abnormalities, although diagnosis is not always straight-forward and may require CT or MRI. In many instances, a characteristic radiologic appearance on plain film, along with age of patient, location of the lesion(s) and presenting symptoms, helps differentiate benign from malignant lesions. Benign lesions, including those in CLA, typically demonstrate bone destruction with well-demarcated margins. However, multiple myeloma and metastases must be evaluated for in adults, particularly when older than 40 years of age. In children, the differential diagnoses for multiple sharply defined osteolytic lesions is narrower and includes LCH and multiple enchondromas. Other benign lesions that are generally singular in nature, such as non-ossifying fibroma, chondroblastoma, chondromyxoid fibroma, osteoid osteoma, giant cell tumor, unicameral and aneurysmal bone cysts, also appear radiolucent on plain film but can typically be differentiated from CLA on MRI or CT imaging, if not by plain film. In children with CLA, bone lesions are frequently found incidentally or with pathologic fractures. When bone pain, laboratory abnormalities, or systemic signs and symptoms are present, metastatic neuroblastoma, Ewing's sarcoma, acute leukemia and osteomyelitis must also be considered. Chronic pain is a frequent presentation in adolescents and adults with CLA.

\subsubsection{Management Considerations}

Respiratory issues secondary to pleural effusion represent a frequent presentation of individuals with GLA. Although the exact incidence is unclear, pleural effusions in GLA appear to be common with an approximate prevalence of 40-70\% [5, 22]. Historically, thoracic involvement in GLA portended significant morbidity and poor prognosis, particularly in children [23]. One retrospective study of 69 children with CLA (35 GLA, 9 KLA, and 41 GSD) reported an overall mortality rate of $20 \%$ in patients with thoracic disease [5]. Of those 35 patients with GLA, 25 (71\%) had thoracic lesions and 10 died (28\%) over a 7-year period. As in the case of our patient, pleural and/or pericardial effusions are known to develop or worsen with illness, including common viral infections. Individuals with GLA are also at risk for worsening effusions during times of growth and hormonal surges, such as puberty and pregnancy.

Until recently, medical treatments were limited to therapies such as steroids, interferon, and chemotherapeutic agents which produced variable outcomes. The discovery of the beneficial effects of mTOR inhibition in LM has led to the use of sirolimus to control and improve disease complications in patients with GLA. In a retrospective study of 18 patients with complex lymphatic anomalies (13 GLA, 5 GSD) treated with sirolimus, 5 of 6 (83\%) patients with GLA had improvement or complete resolution of their pleural effusions. Two of $3(67 \%)$ GLA patients had complete resolution of their pericardial effusions. No GLA patients had worsening of pericardial or pleural effusions while on sirolimus treatment. Additionally, most patients with effusions also experienced improvement in one or more associated complications such as respiratory symptoms, functional impairment, and quality-of-life. Importantly, no deaths occurred over the 7.5-year study period [22]. Recent discovery of somatic PIK3CA genetic mutations in GLA also suggests a therapeutic role for PIK3CA inhibitors [6].

In our patient's case, chest tube placement and pleural fluid drainage was indicated because of cardiorespiratory compromise. If the patient is asymptomatic or has tolerable mild symptoms, drainage is not necessary, even when the effusion is large. From our clinical experience, sirolimus also helps decrease the amount of pleural fluid output while the chest tube is in place and decreases risk of fluid re-accumulation when the drain is removed. 


\subsection{Case 3: Kaposiform Lymphangiomatosis}

A 7-year-old girl with "chronic ITP", diagnosed at an outside institution, presented with abdominal pain and epistaxis and was found to have splenomegaly, moderate thrombocytopenia, and small amount of ascites on ultrasound. She developed worsening ascites and a rapid decline in hemoglobin and platelets as well as PT and PTT prolongation. Paracentesis was performed revealing grossly bloody peritoneal fluid. Her hematologic abnormalities were initially thought to be due to ITP in combination with acute consumption of platelets and coagulation factors. She was subsequently given packed red blood cell (RBC), fresh frozen plasma and multiple platelet transfusions. Her platelet count and fibrinogen level were noted to initially worsen and then gradually improve. The patient recovered from this event but developed several more episodes of painful ascites, which were likely hemorrhagic, although repeat paracentesis was not performed. Given her presumed diagnosis of ITP, she underwent splenectomy. Pathology revealed diffuse infiltration of her spleen with KLA (Figure 3A-B) .

\subsubsection{Diagnostic Considerations}

KLA is a rare yet very aggressive disease in which early diagnosis and timely treatment are critical. KLA has only been recognized as a distinct clinical entity since 2013 and unfortunately, is often misdiagnosed [7]. Like GLA and GSD, KLA is inaccurately referred as "lymphangiomatosis" but coagulopathy is the hallmark of KLA. In patients with unexplainable hypofibrinogenemia, thrombocytopenia, and bleeding, particularly if hemorrhagic ascites, pleural effusion, and/or pericardial effusion are present, KLA must be considered. KLA commonly involves bones and viscera and infiltrates into the thoracic and abdominal cavities. When located in superficial soft tissues, KLA appears as non-discrete red-purple purpuric lesions. The most common presenting clinical features of KLA are bleeding and respiratory symptoms such as cough and dyspnea [7].

Imaging is important for diagnosis of KLA, particularly since biopsy is often unable to be performed safely. MRI imaging of the chest, abdomen, pelvis, and total spine. High resolution chest CT may provide additional information for lung or mediastinal involvement. Pleural and pericardial effusions, along with LM involvement of the thoracic cavity, spleen and bones are common. Similar to GLA, the osteolytic bone lesions are cortex-sparing and involve multiple non-contiguous bones, most commonly the vertebrae [7]. On imaging, KLA of the soft tissue appears as an infiltrative abnormality on fluid-weighted MRI sequences, frequently with stranding of the adjacent subcutaneous fat [24]. On contrast-enhanced CT, KLA appears as infiltrative low-density soft-tissue thickening or mass, and effusions are of low attenuation. KLA is heterogeneously hyperintense on fluid-weighted MRI sequences, with moderate to intense post-contrast enhancement and follows the lymphatic distribution along bronchovascular bundles (Figure 4A). Soft tissue thickening around the blood vessels and airways in the anterior mediastinum and interlobular septal thickening are also frequent findings (Figure 4B) . In viscera, KLA appears as hyperintense round lesions on fluid-weighted MRI sequences and hypodense lesions on CT. Enhancing infiltrative soft tissue is also frequently seen in the retroperitoneum and abdomen [24]. Echocardiogram is also indicated to evaluate for pericardial effusion.

If a soft tissue component is present, biopsy may be possible, depending on severity of coagulopathy. If KLA is confirmed by pathology, NRAS testing of the tissue specimen should be considered to guide therapy [10]. In our patient, a somatic $N R A S$ Q61R mutation with an allele frequency of approximately $5 \%$ was found in her splenic tissue.

\subsubsection{Management Considerations}

Without treatment, KLA commonly progresses and causes hemorrhage, effusions, cardiorespiratory compromise, multiorgan failure or even death. Unfortunately, there is no cure for KLA. Goal of therapy is to halt disease progression, control coagulopathy, manage symptoms and prevent complications. Currently, no universal guidelines exist for the treatment of KLA. KLA typically cannot be completely removed with surgery because of its infiltrative and multifocal nature and should be avoided given the high bleeding risk. Various medical therapies such as steroids, single or multiple chemotherapy agents, interferon, and sirolimus, have been used [9, 25-27]. Often used in combination as 2-drug or 3-drug regimens, oral sirolimus, intravenous or oral steroids, and vincristine, have become the preferred agents in the initial treatment of KLA [16]. Refer 
to Table 2 for suggested treatment regimen(s).

Bleeding complications are common in patients with KLA, but blood product replacement must be performed with caution. Platelet transfusions can worsen the underlying coagulopathy and oftentimes, does not improve platelet counts. Platelet transfusions should only be given for active, uncontrolled bleeding or immediately prior to or during a procedure with more than low risk for bleeding [27]. Fresh frozen plasma (FFP) or cryoprecipitate is recommended for hypofibrinogenemia less than $100 \mathrm{mg} / \mathrm{dL}$. Prior to any procedure with more than minimal to low risk of bleeding and in patients with active bleeding, fibrinogen should be corrected to greater than $150 \mathrm{mg} / \mathrm{dL}$. RBC transfusions do not have any negative effect on KLA, so should be given to maintain oxygenation, perfusion and symptomatic relief.

In our patient, platelet transfusions worsened her hypofibrinogenemia and failed to improve her thrombocytopenia. This is an expected phenomenon occurring because of increased platelet trapping in the KLA lesions and subsequent activation of the coagulation cascade. She was initially started on sirolimus but due to intolerable side effects was switched to the MEK inhibitor, trametinib. Her hematologic parameters normalized on sirolimus, and her disease remains controlled on trametinib.

\section{CONCLUSION}

CLA are debilitating chronic diseases that can be life-threatening. Given the progressive nature of these conditions, early and accurate diagnosis is essential for optimal management, which typically requires an interdisciplinary and multimodal approach. Although CLA do not have a cure, recent clinical and scientific discoveries have enhanced our knowledge of the genetics and pathophysiology of these conditions and changed the treatment paradigm, now favoring medical management to control disease progression and complications as well as improve symptoms and QOL.

\section{CONFLICT OF INTEREST STATEMENT}

The authors declare no conflicts of interest.

\section{ACKNOWLEDGMENTS}

The authors are grateful to the staff of the Cincinnati Children's Research Foundation and Vascular Anomalies Center at Texas Children's Hospital, and to the patients and families for participation in research to improve knowledge and understanding of these disorders.

\section{REFERENCES}

1. Trenor, C.C., 3rd and G. Chaudry, Complex lymphatic anomalies.Semin Pediatr Surg, 2014. 23 (4): p. 186-90.

2. Lala, S., et al., Gorham-Stout disease and generalized lymphatic anomaly-clinical, radiologic, and histologic differentiation. Skeletal Radiol, 2013. 42 (7): p. 917-24.

3. de Villiers, J.F. and W.R. Stevens, Case 203: Gorham disease.Radiology, 2014. 270 (3): p. 931-5.

4. North, P.E., Pediatric Vascular Tumors and Malformations. Surg Pathol Clin, 2010. 3 (3): p. 455-94.

5. Ozeki, M., et al., Clinical Features and Prognosis of Generalized Lymphatic Anomaly, Kaposiform Lymphangiomatosis, and Gorham-Stout Disease. Pediatr Blood Cancer, 2016. 63 (5): p. 832-8.

6. Rodriguez-Laguna, L., et al., Somatic activating mutations in PIK3CA cause generalized lymphatic anomaly. J Exp Med, 2019.216 (2): p. 407-418.

7. Croteau, S.E., et al., Kaposiform lymphangiomatosis: a distinct aggressive lymphatic anomaly. J Pediatr, 2014. 164 (2): p. 383-8.

8. Adams, D.M. and K.W. Ricci, Vascular Anomalies: Diagnosis of Complicated Anomalies and New Medical Treatment Options. Hematol Oncol Clin North Am, 2019. 33 (3): p. 455-470. 
9. Ji, Y., et al., Kaposiform lymphangiomatosis and kaposiform hemangioendothelioma: similarities and differences. Orphanet J Rare Dis, 2019. 14 (1): p. 165.

10. Barclay, S.F., et al., A somatic activating NRAS variant associated with kaposiform lymphangiomatosis. Genet Med, 2019.21 (7): p. 1517-1524.

11. Krishnamurthy, R., et al., Imaging the central conducting lymphatics: initial experience with dynamic MR lymphangiography.Radiology, 2015. 274 (3): p. 871-8.

12. Dori, Y., M.M. Zviman, and M. Itkin, Dynamic contrast-enhanced MR lymphangiography: feasibility study in swine. Radiology, 2014.273 (2): p. 410-6.

13. Li, D., et al., Pathogenic variant in EPHB 4 results in central conducting lymphatic anomaly. Hum Mol Genet, 2018. 27 (18): p. 3233-3245.

14. Iacobas, I., et al., Multidisciplinary guidelines for initial evaluation of complicated lymphatic anomaliesexpert opinion consensus.Pediatr Blood Cancer, 2020. 67 (1): p. e28036.

15. Alomari, M.C., R; Kerr, Cindy; O'Hare, M; Padua, H; Chaudry, G; Chewning R; Alomari, A, Chylothorax Following Rib Biopsy in Generalized Lymphatic Anomaly in 22nd International Workshop of the International Society for the Study of Vascular Anomalies . 2018: Amsterdam, Netherlands.

16. Adams, D.M., et al., Vascular anomaly cases for the pediatric hematologist oncologists-An interdisciplinary review. Pediatr Blood Cancer, 2018. 65 (1).

17. Simon, F., et al., Management of Gorham Stout disease with skull-base defects: Case series of six children and literature review.Int J Pediatr Otorhinolaryngol, 2019. 124 : p. 152-156.

18. Evans, D.A., et al., Lymphangiomatosis of Skull Manifesting with Recurrent Meningitis and Cerebrospinal Fluid Otorrhea.Otolaryngology-Head and Neck Surgery, 1990. 103 (4): p. 642-646.

19. Nazarian, G.K., S.S. Gebarski, and J.K. Niparko, Cranial lymphangiomatosis causing CSF otorrhea and recurrent meningitis: CT features. J Comput Assist Tomogr, 1990. 14 (1): p. 121-3.

20. Venkatramani, R., et al., Gorham's disease and diffuse lymphangiomatosis in children and adolescents. Pediatr Blood Cancer, 2011. 56 (4): p. 667-70.

21. Cramer, S.L., et al., Gorham-Stout Disease Successfully Treated With Sirolimus and Zoledronic Acid Therapy. J Pediatr Hematol Oncol, 2016. 38 (3): p. e129-32.

22. Ricci, K.W., et al., Efficacy of systemic sirolimus in the treatment of generalized lymphatic anomaly and Gorham-Stout disease.Pediatr Blood Cancer, 2019. 66 (5): p. e27614.

23. Alvarez, O.A., I. Kjellin, and C.W. Zuppan, Thoracic lymphangiomatosis in a child. J Pediatr Hematol Oncol, 2004.26 (2): p. 136-41.

24. Goyal, P., et al., Imaging features of kaposiform lymphangiomatosis. Pediatr Radiol, 2016. 46 (9): p. 1282-90.

25. Fernandes, V.M., et al., Kaposiform lymphangiomatosis: unifying features of a heterogeneous disorder. Pediatr Blood Cancer, 2015. 62 (5): p. 901-4.

26. Hammill, A.M., et al., Sirolimus for the treatment of complicated vascular anomalies in children. Pediatr Blood Cancer, 2011.57 (6): p. 1018-24.

27. Adams, D.M., Special considerations in vascular anomalies: hematologic management. Clin Plast Surg, 2011. 38 (1): p. 153-60.

28. Mizuno, T., et al., Developmental pharmacokinetics of sirolimus: Implications for precision dosing in neonates and infants with complicated vascular anomalies. Pediatr Blood Cancer, 2017.64 (8). 


\section{FIGURE 1}
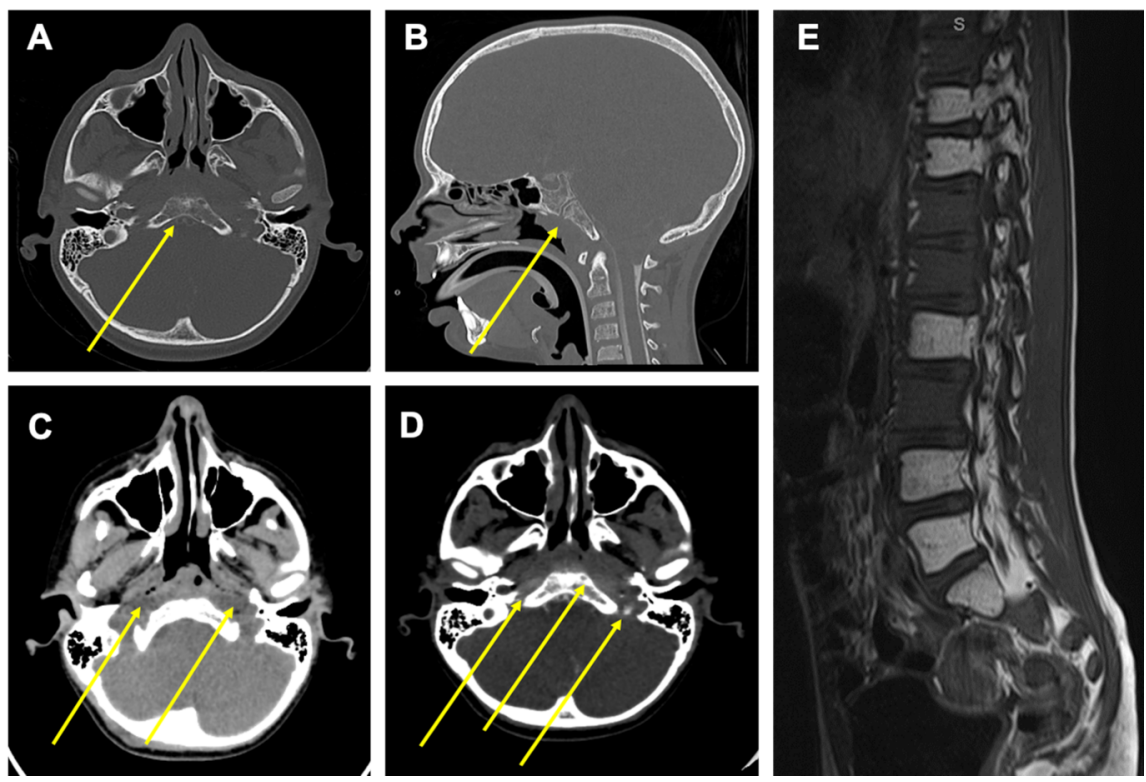

Figure 1. Gorham-Stout Disease affecting skull base in a child and causing recurrent meningitis. (A) Axial view and (B) sagittal view of bone window showing the lymphatic malformation in central skull base (arrows). Notice the bone is heterogeneous with lysis especially at cortex. (C) Pre-contrast soft tissue window for baseline. Notice the hypodense area next to central skull base (arrows). (D) Post-contrast myelogram. Faint hyper-density in soft tissues next to the central skull base is the leaking contrast from the porous bone (arrows). (E) Spine involvement. Diffuse T1 shortening and T2 prolongation on vertebrae T10-T11, L2 and L4-S1. Majority of these vertebral bodies are nearly completely affected, some with both the anterior and posterior elements involvement.

\section{FIGURE 2}

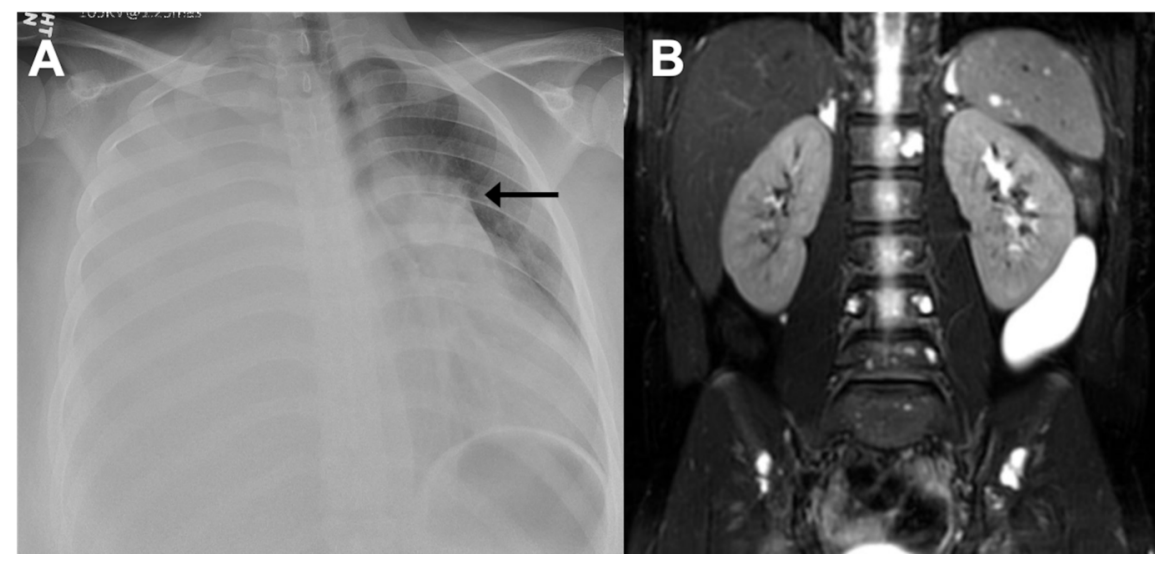

Figure 2. Individual with GLA. (A) Chest x-ray demonstrating large left-sided pleural effusion causing midline shift. Black arrow indicates an osteolytic bone lesion of the rib. (B) MRI showing multiple enhancing lymphatic lesions with the spleen, vertebrae, and pelvic bone on T2-weight sequence. Lymphatic cyst of the left retroperitoneum also identified. 


\section{FIGURE 3}

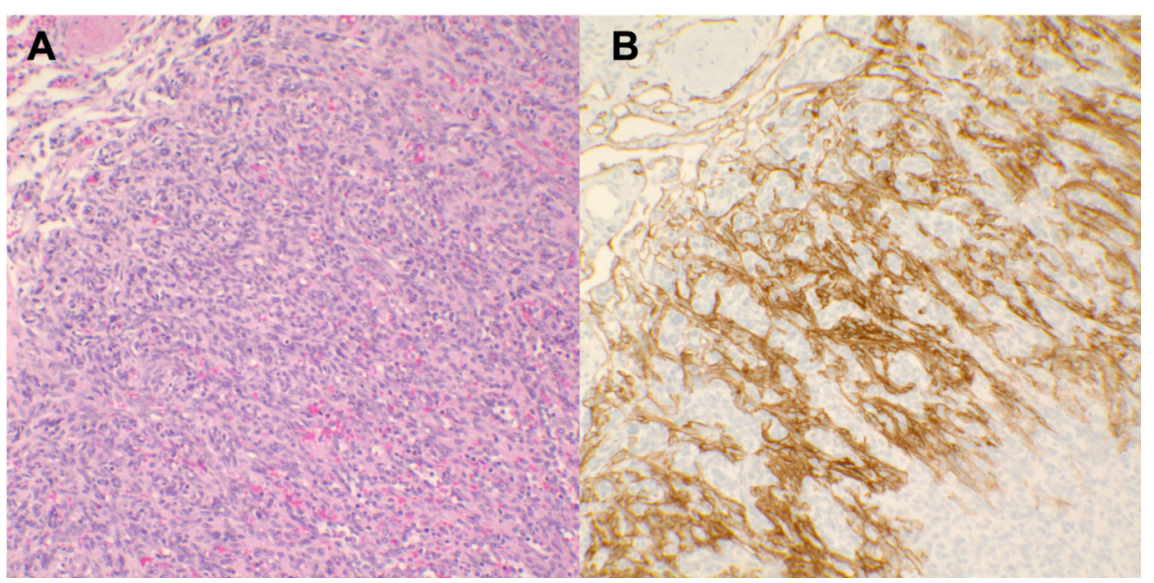

Figure 3. Pathology of spleen of a patient with KLA. (A) H Hematoxylin-eosin staining shows dilated lymphatic channels and diffuse infiltrative KLA component in "sheet-like" distribution with characteristic kaposiform or spindle endothelial cells. (B) Kaposiform cells stain positively for D2-40.

\section{FIGURE 4}

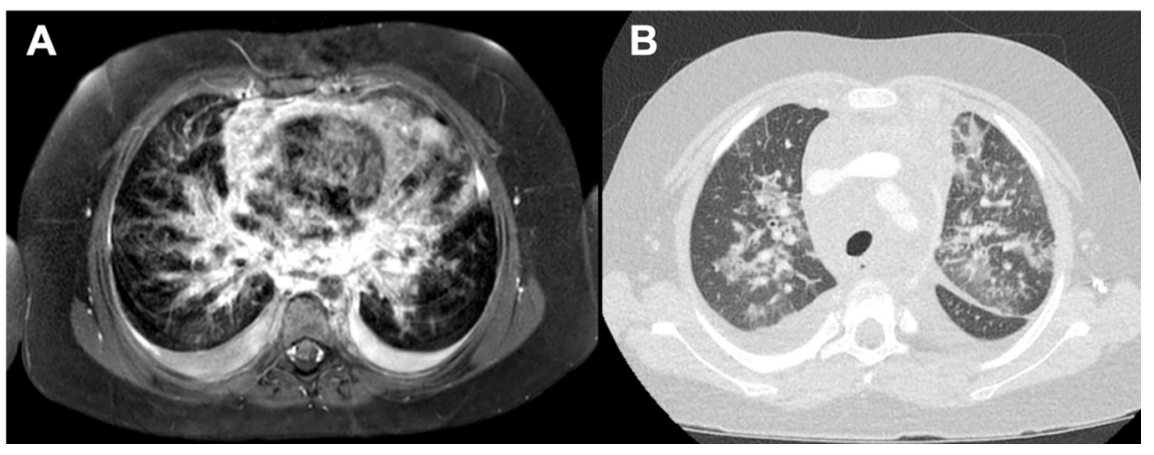

Figure 4. Imaging of pulmonary KLA. (A) MRI T2-weighted imaging reveals infiltrative hyperintense signal throughout the anterior and middle mediastinum as well as diffuse peribronchovascular signal abnormality. (B) High-resolution CT demonstrates intralobar septal thickening, ground glass opacities, and bilateral pleural effusions.

TABLE 1: Summary of clinical and histopathologic features of complex lymphatic anomalies

\begin{tabular}{lll}
\hline COMPLEX LYMPHATIC & & HISTOPATHOLOGY/ \\
ANOMALY & CLINICAL MANIFESTATIONS & MOLECULAR GENETICS \\
\hline Gorham-Stout Disease (GSD) & "Vanishing" bone diseaseà & PROX-1, D2-40 positive \\
& pathologic fractures, functional & abnormal lymphatics with \\
& issues, CSF leaks, meningitis, & increased osteoclast activity \\
& spinal instability, deformity & and destruction of bone cortex \\
& Effusions Chronic pain & Mutation: unknown
\end{tabular}




\begin{tabular}{|c|c|c|}
\hline $\begin{array}{l}\text { COMPLEX LYMPHATIC } \\
\text { ANOMALY }\end{array}$ & CLINICAL MANIFESTATIONS & $\begin{array}{l}\text { HISTOPATHOLOGY/ } \\
\text { MOLECULAR GENETICS }\end{array}$ \\
\hline $\begin{array}{l}\text { Generalized Lymphatic } \\
\text { Anomaly (GLA) }\end{array}$ & $\begin{array}{l}\text { Multisite lymphatic } \\
\text { malformation of soft tissue, } \\
\text { viscera (spleen, and liver } \\
\text { common) and bones- bone } \\
\text { lesions are multiple and } \\
\text { non-contiguous Effusions } \\
\text { Chronic Pain }\end{array}$ & $\begin{array}{l}\text { Increased number of dilated } \\
\text { anastomosing lymphatic } \\
\text { channels, lined by endothelial } \\
\text { cells, stains PROX-1 and D2-40 } \\
\text { positive Mutation: PIK3CA }\end{array}$ \\
\hline $\begin{array}{l}\text { Central Conducting Lymphatic } \\
\text { Anomaly (CCLA) }\end{array}$ & $\begin{array}{l}\text { Enlarged lymphatic } \\
\text { channels/cysts in abdomen } \\
\text { and/or thorax Reflux of } \\
\text { lymphatic fluid à pleural and } \\
\text { pericardial effusions, ascites, } \\
\text { massive edema Protein loss } \\
\text { Recurrent infections Organ } \\
\text { dysfunction }\end{array}$ & $\begin{array}{l}\text { Dilated lymphatic } \\
\text { channels-vessels aren't } \\
\text { malformed but are } \\
\text { dysfunctional or distally } \\
\text { obstructed Mutation: EPHB4 }\end{array}$ \\
\hline
\end{tabular}

Kaposiform Lymphangiomatosis (KLA) Multifocal lymphatic anomaly that often involves thorax, bones, viscera Progress

TABLE 2: Medication treatment options and recommendations by complex lymphatic anomaly

\begin{tabular}{|c|c|c|c|c|c|c|}
\hline MEDICATION & INDICATION & DIAGNOSES & $\begin{array}{l}\text { INITIAL } \\
\text { DOSE/ } \\
\text { REGIMEN }\end{array}$ & $\begin{array}{l}\text { LENGTH } \\
\text { OF } \\
\text { THERAPY }\end{array}$ & $\begin{array}{l}\text { POTENTIAL } \\
\text { COMPLICATIO }\end{array}$ & $\begin{array}{l}\text { POTENTIAL } \\
\text { NSQMPLICATIO }\end{array}$ \\
\hline$\overline{\text { Sirolimus }}$ & $\begin{array}{l}\text { Bone lytic } \\
\text { lesions } \\
\text { Effusions LM } \\
\text { engorgement } \\
\text { Pain }\end{array}$ & $\begin{array}{l}\text { GLA GSD } \\
\text { KLA } \\
\text { CCLA++ }\end{array}$ & $\begin{array}{l}0.8 \mathrm{mg} / \mathrm{m}^{2} / \text { dose } \\
\text { PO every } 12 \\
\text { hours* Trough } \\
\text { target level: } \\
\text { 8-12ng/mL }\end{array}$ & $\begin{array}{l}\text { Indefinite OR } \\
\text { transition to } \\
\text { other targeted } \\
\text { therapy }\end{array}$ & $\begin{array}{l}\text { Oral mucositis } \\
\text { High } \\
\text { triglycerides } \\
\text { Immunosup- } \\
\text { pression } \\
\text { (consider PJP } \\
\text { prophylaxis) }\end{array}$ & $\begin{array}{l}\text { Oral mucositis } \\
\text { High } \\
\text { triglycerides } \\
\text { Immunosup- } \\
\text { pression } \\
\text { (consider PJP } \\
\text { prophylaxis) }\end{array}$ \\
\hline $\begin{array}{l}\text { Interferon } \\
\text { alpha } 2 \mathrm{~b}\end{array}$ & $\begin{array}{l}\text { Bone lytic } \\
\text { lesions } \\
\text { Effusions }\end{array}$ & $\begin{array}{l}\text { GLA GSD } \\
\text { KLA }\end{array}$ & $\begin{array}{l}\text { Pegylated } \\
\text { form: } \\
1.5 \mathrm{mcg} / \mathrm{kg} / \mathrm{dose} \\
\text { subQ weekly }\end{array}$ & $\begin{array}{l}\text { Indefinite (but } \\
\text { may be limited } \\
\text { due to } \\
\text { complications) }\end{array}$ & $\begin{array}{l}\text { Flu-like } \\
\text { symptoms } \\
\text { with } \\
\text { administration } \\
\text { Hypothyroidism }\end{array}$ & \\
\hline Vincristine & $\begin{array}{l}\text { Coagulopathy } \\
\text { Effusions }\end{array}$ & KLA & $\begin{array}{l}\text { >10kg: } \\
1.5 \mathrm{mg} / \mathrm{m}^{2} / \text { dose } \\
\text { IV weekly } \\
\text { initially, } \max \\
2 \mathrm{mg} / \text { dose }[?] 10 \\
\mathrm{~kg}: \\
0.05 \mathrm{mg} / \mathrm{kg} / \text { dose }\end{array}$ & $\begin{array}{l}\text { Taper off as } \\
\text { coagulopathy } \\
\text { improves }\end{array}$ & $\begin{array}{l}\text { Peripheral } \\
\text { neuropathy } \\
\text { Constipation } \\
\text { Bone pain } \\
\text { SIADH } \\
\text { Extravasation } \\
\text { risk Hair loss }\end{array}$ & $\begin{array}{l}\text { Peripheral } \\
\text { neuropathy } \\
\text { Constipation } \\
\text { Bone pain } \\
\text { SIADH } \\
\text { Extravasation } \\
\text { risk Hair loss }\end{array}$ \\
\hline
\end{tabular}




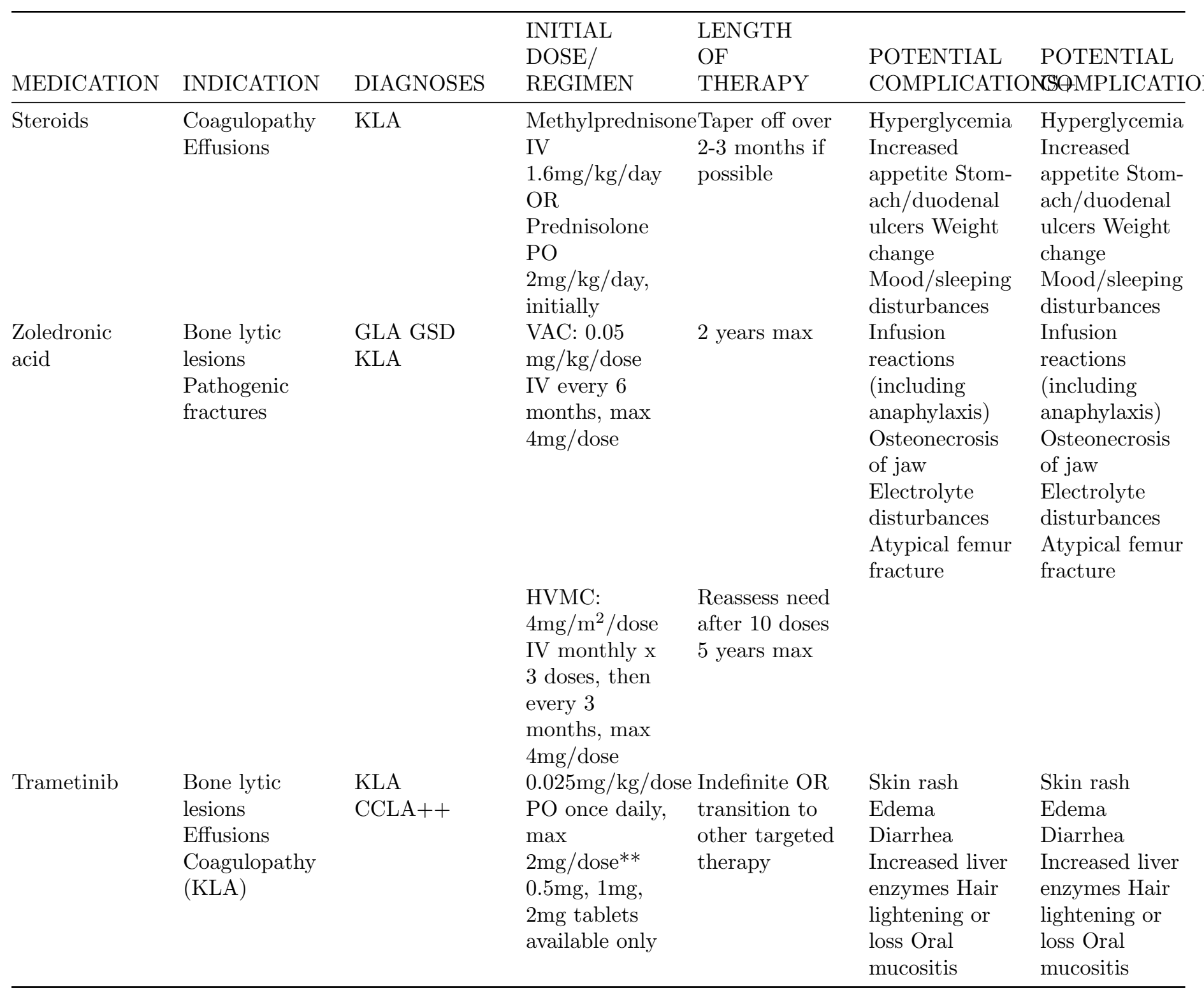

Abbreviations : CCLA, central conducting lymphatics anomaly; HVMC, Hemangioma and Vascular Malformation Center at Cincinnati Children's Hospital Medical Center; GLA, generalized lymphatic anomaly; GSD, Gorham-Stout Disease; IV, intravenously; KLA, kaposiform lymphangiomatosis; LM, lymphatic malformation; PJP, Pneumocystis jirovecii pneumonia; PO, per oral; SIADH, syndrome of inappropriate antidiuretic hormone secretion; subQ, subcutaneously; VAC, Vascular Anomalies Center at Texas Children's Cancer and Hematology Centers.

+ Common and notable lesser common complications listed. This is not an extensive list of all potential side effects.

++ Response to disease-modifying agents such as sirolimus and trametinib for CCLA is unclear. EPHB4 mutation recently found in CCLA suggests there may be a role for mTOR or MAPK/MEK inhibition.

*Dosing for infants and young children is different due to metabolism differences. Recommended dosing for infants and young children under 2 years of age is based on pharmacokinetic studies by Mizuno et al. [28] 
**Suggested dosing based on clinical trials of trametinib use in children with plexiform neurofibromas and gliomas.

\section{Hosted file}

TABLE 1.pdf available at https://authorea.com/users/377907/articles/494538-how-we-approachthe-diagnosis-and-management-of-complex-lymphatic-anomalies

\section{Hosted file}

TABLE 2.pdf available at https://authorea.com/users/377907/articles/494538-how-we-approachthe-diagnosis-and-management-of-complex-lymphatic-anomalies
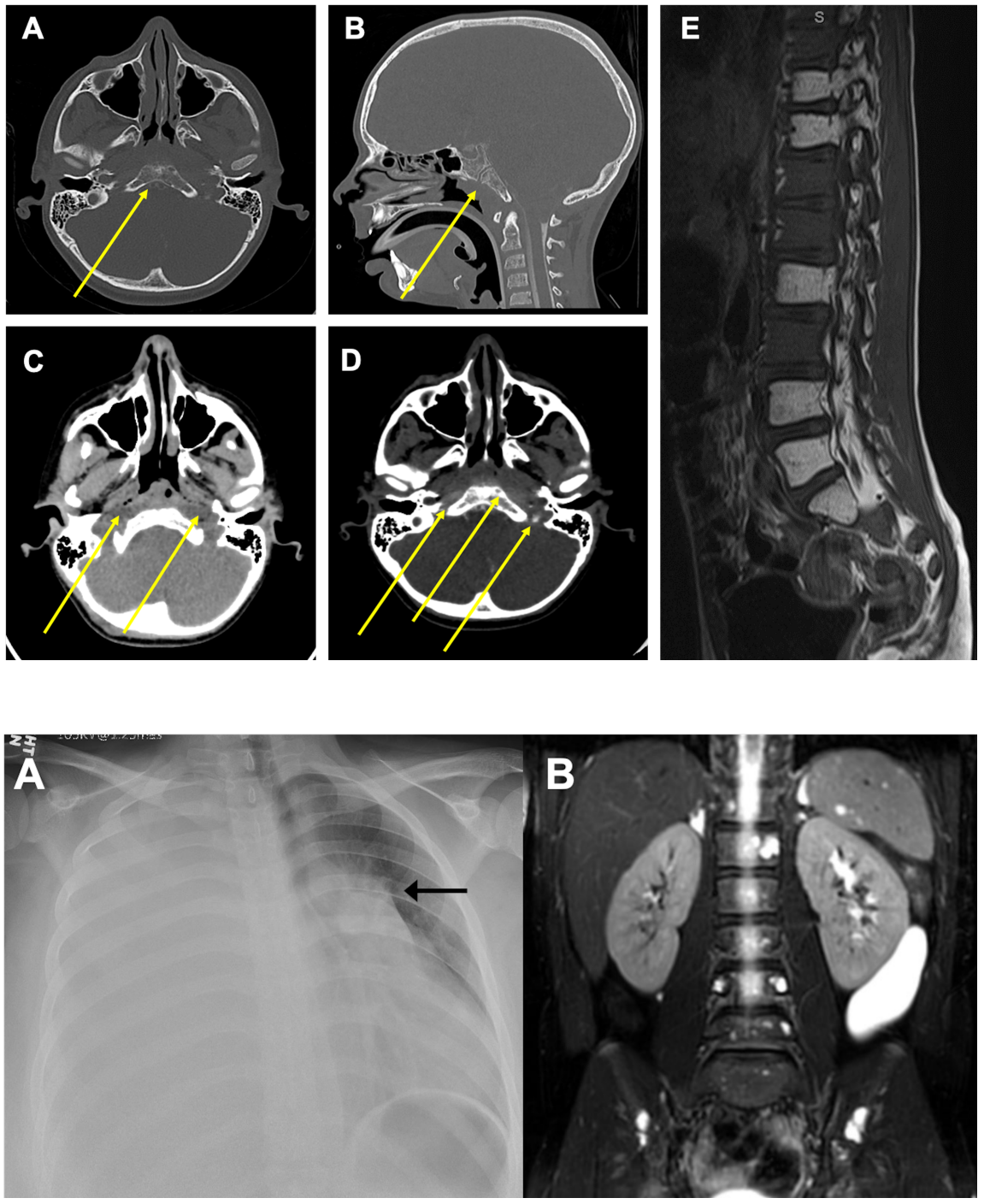

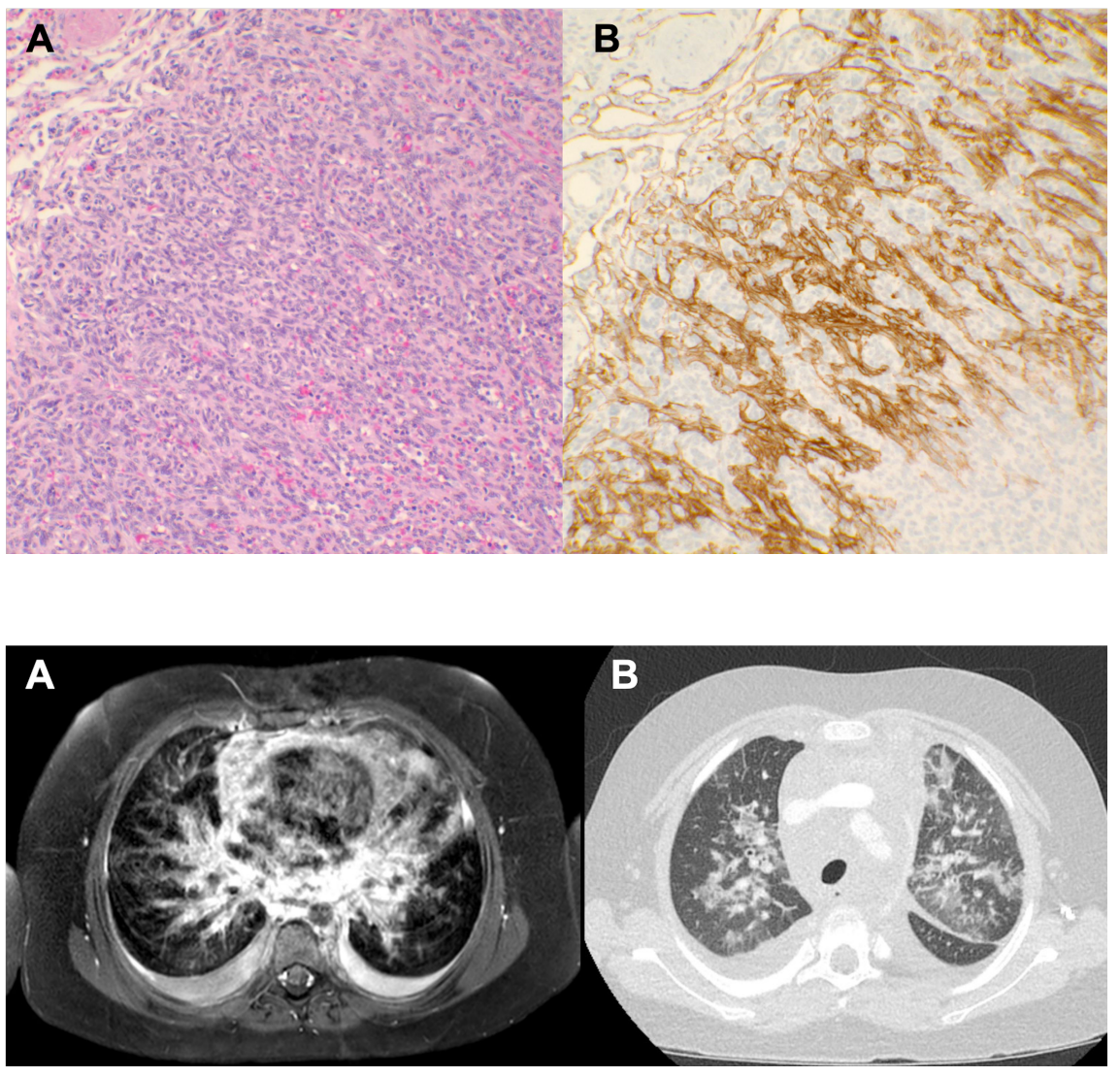